Itinéraires Itinéraires

Littérature, textes, cultures

2016-1 | 2016

Écrire et créer avec les villes en mouvement

\title{
Histoire et fiction dans Beyrouth Adrénaline (2008) de Hala Ghosn
}

History and Fiction in Beyrouth Adrénaline (2008) by Hala Ghosn

Isabelle Bernard

\section{OpenEdition}

Journals

Édition électronique

URL : http://journals.openedition.org/itineraires/3327

DOI : $10.4000 /$ itineraires.3327

ISSN : 2427-920X

Éditeur

Pléiade

Référence électronique

Isabelle Bernard, «Histoire et fiction dans Beyrouth Adrénaline (2008) de Hala Ghosn », Itinéraires [En ligne], 2016-1 | 2016, mis en ligne le 01 décembre 2016, consulté le 20 avril 2019. URL : http:// journals.openedition.org/itineraires/3327 ; DOI : 10.4000/itineraires.3327

Ce document a été généré automatiquement le 20 avril 2019.

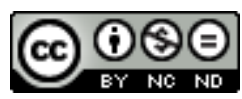

Itinéraires est mis à disposition selon les termes de la licence Creative Commons Attribution - Pas d'Utilisation Commerciale - Pas de Modification 4.0 International. 


\title{
Histoire et fiction dans Beyrouth Adrénaline (2008) de Hala Ghosn
}

\author{
History and Fiction in Beyrouth Adrénaline (2008) by Hala Ghosn
}

Isabelle Bernard

« On nomme littérature la fragilité de l'histoire. »

(Boucheron 2011:41)

1 Depuis une trentaine d'années, la littérature française se donne de plus en plus pour objet d'appréhender le réel et l'histoire, en particulier les périodes tumultueuses $\mathrm{du} \mathrm{xx}^{\mathrm{e}}$ siècle, à travers ses approches de la mémoire et de l'oubli, individuels ou collectifs. Si la porosité des frontières entre les deux disciplines majeures des sciences humaines n'est pas neuve, la volonté des auteurs dramatiques de répondre, pour eux-mêmes et leurs spectateurs, à cette « demande et cet appel, venus des profondeurs sociales, à un renouvellement dans l'approche et l'appréhension du passé » (Nora 2011 : 12) n'a de cesse de se renouveler. Le discours intime, introspectif, et le traitement de la mémoire sont devenus des éléments formels récurrents de la dramaturgie francophone contemporaine. Cette propension de la fiction $^{1}$ à servir autant que se servir de l'histoire est devenue une pratique artistique dominante (Compagnon $2011:$ 62), ce que le succès du quatuor Le Sang des promesses de Wajdi Mouawad, par exemple, excellant à exprimer la souffrance de l'exil, de la guerre et de la perte, indique ${ }^{2}$ également :

[...] au-delà des clivages idéologiques, esthétiques et dramaturgiques, deux constantes apparaissent dans l'art du théâtre depuis les années 1980 : le désir de lier le moi et le monde, d'articuler l'intime et le collectif, et le parti pris de renouveler la diction et la représentation du réel, interrogeant ainsi l'essence même du théâtre. (Évrard 2008 : 500)

2 En scrutant le fonctionnement intime de Beyrouth adrénaline, une pièce centrée sur la guerre fratricide qui a défiguré le Liban entre 1975 et 1992 et plus sporadiquement jusqu'en 2006, nous proposons de montrer que l'écrit théâtral s'est emparé des «symptômes littéraires de nos interrogations et nos retours sur les fondations de notre modernité » (Gengembre 2011 : 131). Nous présenterons les modalités d'écriture de cette œuvre qui s'ancre dans le parcours intime de son auteure; nous soulignerons ensuite la 
pratique de Ghosn, qui s'inscrit dans la nébuleuse des écritures de plateau actuelles; enfin, nous expliciterons les défis anthropologiques soulevés par ce texte saisi par l'histoire contemporaine.

\section{Beyrouth, 1986 ou Un singulier modus scribendi}

3 Née en 1976, Hala Ghosn a subi l'exil quelques mois après sa naissance lorsqu'en 1977 ses parents ont fui la guerre qui faisait rage au Liban. Alors qu'une partie de sa famille est demeurée à Beyrouth, elle a grandi en France, où elle a découvert le théâtre qui est très rapidement devenu sa passion en tant que comédienne, auteure dramatique et metteure en scène... Dès 1994, elle a en effet suivi une formation à l'Atelier international de théâtre de Paris puis, en 1997, à l'Académie du théâtre de l'Union au Centre dramatique national du Limousin. Ghosn a fait ses premiers pas sur scène en tant que comédienne et, à partir de 2002, est passée à la mise en scène et à l'écriture : elle a coécrit Duo pour trois et Les Angelots avec Céline Garnavault et Arnaud Chéron. Avec Jalie Barcilon, elle a expérimenté l'écriture de plateau au sein du collectif La Poursuite-Makizart et a notamment publié Beyrouth adrénaline (2006) et Apprivoiser la panthère (2010).

4 L'artiste franco-libanaise axe son écriture autour de thématiques interculturelles, multiconfessionnelles, pacifiques et identitaires : elle incarne elle-même un tiraillement dolent entre l'Orient et l'Occident qu'elle transforme en un devoir de mémoire. Ses productions les plus récentes, Une cigarette au sporting : petite géopolitique en maillot de bain (2011), Apprivoiser la panthère, Mon petit guide en Croatie (2013) ou Les Primitifs (2014) se lisent comme des appels au dialogue et au respect des êtres et de leurs libertés, par-delà leurs différences, leurs choix et leurs appartenances: tous témoignent d'une profonde réflexion sur le déclenchement des conflits fratricides, sur les passions que suscitent les nationalismes ; ils disent les désirs, les haines, les intolérances du quotidien, mais aussi la liberté revendiquée et la paix toujours précaires face à l'oppression.

Créée en $2006^{3}$, Beyrouth adrénaline se compose de douze tableaux écrits au plateau par Hala Ghosn et Jalie Barcilon avec les acteurs de la compagnie La Poursuite-Makizart. Elle explore le traumatisme familial de Ghosn, cette déchirure tragique due au suicide d'une nation plusieurs fois millénaire. Pour l'écrivaine qui s'est longuement interrogée sur le phénomène des replis identitaires et des communautarismes, il s'agit de témoigner de l'expérience tragique qui est la sienne et celle de ses concitoyens nés au moment du déclenchement du conflit. Son parti pris est de faire connaître l'expérience plurielle des Beyrouthins, de ceux qui ont fui pour s'installer en France et qui n'auront connu le conflit que par médias interposés, et de ceux qui sont restés. «Tous ces personnages sont autant de façons différentes de voir et de dire le conflit libanais. Certains l'acceptent et d'autres l'occultent. Certains le vivent, d'autres l'imaginent» (Ghosn 2008: 9). Convaincue que tous ont souffert qu'ils aient ou non vécu dans la crainte des bombardements et sous les tirs des snipers, elle montre les effets pathogènes de la guerre civile à travers le déchirement intérieur de tous les Libanais, leur désarroi et leurs désirs profonds.

Aussi la pièce met-elle en scène une fratrie éclatée: en 1986, à Paris, il y a Zyad, conférencier spécialiste du Moyen-Orient, et sa sœur Mona, 15 ans. Tiraillée par sa double nationalité, l'adolescente idéalise le Liban de sa petite enfance : c'est du reste dans la plus longue réplique de la pièce qu'elle dépeint « la perle de l'Orient, sublimée par Lamartine et Gérard de Nerval» (Ghosn $2008: 68$ ), ses paysages magnifiques et contrastés, l'accueil 
généreux de ses habitants, le tout effacé par la guerre (Ghosn 2008:34-35); elle milite dans les milieux estudiantins et désire connaître son pays en dépit du chaos qui y règne, alors que son aîné qu'elle juge passablement lâche (Ghosn 2008: 35) a totalement intellectualisé le conflit; afin d'estomper sa culpabilité d'être parti, il tente quant à lui d'en cerner les tenants et aboutissements et donne dans la séquence finale une conférence (Ghosn 2008: 68-72) en forme d'historique qui révèle plus que ses connaissances une angoisse pathétique et fébrile. Sophie, la petite amie de Zyad, est française. Docteur en psychologie, elle met des mots sur les séquelles du traumatisme vécu par Zyad et Mona et, cherchant sa place dans leur famille, s'interpose lorsqu'ils s'entredéchirent, quitte à complètement s'effacer (Ghosn 2008 : 53).

7 À Beyrouth, il y a Marwan, le frère de Mona et Zyad qui n'a pu quitter le pays, et leur tante Najat. Lui s'entraîne au 400 mètres au cas où la guerre cesserait bientôt (Ghosn 2008 : 29) - et son rêve de victoire dure toute une scène avant de se heurter à la réalité du stade détruit par les bombes (Ghosn 2008: 44-45) -, pendant qu'elle témoigne par ses souvenirs épars d'un pays qui n'existe plus. Dans les propos de la vieille dame, à saisir entre détresse et sénilité, dates et événements se confondent: "Souffrant d'un léger Alzeihmer, elle vacille entre Beyrouth des années quarante et l'époque ottomane" (Ghosn 2008: 9, 41). La confidente de Najat est Sandya, sa bonne sri lankaise ; totalement étrangère au conflit, elle incarne au second plan un autre drame actuel, celui de l'immigration de masse des asiatiques en situation précaire vers les pays du Golfe et le Moyen-Orient. Leur voisine, Rima, est une jeune fonctionnaire du ministère de l'Éducation fiancée à Fadih, un artiste porté disparu depuis trois années; refusant obstinément de quitter Beyrouth (Ghosn 2008: 50), Rima vit dans l'espoir du retour de son aimé avec son frère cadet, Toufic, un adolescent qui, avec l'énergie du désespoir, vient d'intégrer une milice. Il a 16 ans. Beyrouth leur demeure à tous une source intarissable d'adrénaline.

8 L'engagement à vocation politique de Hala Ghosn est largement nourri par ses lectures et ses rencontres, ainsi que par une ouverture aussi franche que décomplexée aux cultures d'autrui. Ainsi, dans Beyrouth adrénaline, la dramaturge a-t-elle fait principalement sienne la pensée d'Amin Maalouf explicitée de son essai Les Identités meurtrières (1998). Parmi d'autres ouvrages de références, cette vaste argumentation qui dépeint l'identité tel un processus mouvant, un échange constant qui se forme dans le dialogisme, lui est un vade mecum, certainement parce que, sur fond de communautarisme religieux et de dissensions politiques, les clivages les plus profonds ayant conduits au conflit armé dans son pays natal étaient identitaires. Sur la base du paradigme de la complexité, l'académicien (né en 1949), lui aussi franco-libanais, analyse à travers l'histoire européenne et moyen-orientale récente comment une situation donnée conduit des hommes coexistant dans la paix à l'hystérie meurtrière, comment l'affirmation de soi et de sa légitime liberté trop souvent mène à la négation de l'autre et de sa culture. Au-delà de tout fatalisme, il étudie avec l'acuité de l'historien les tensions sociales du dernier demi-siècle mais, derrière les faits et la violence, les fanatismes religieux et les extrémismes politiques, il tente bien plus sûrement de cerner l'humain dans sa capacité comme dans son incapacité à faire du "vivre-ensemble» un projet pérenne. Et, en donnant la primauté à l'éducation pour tous et au respect de chacun, il ébauche un programme politique pour l'avenir transeuropéen et méditerranéen. C'est en maintenant des liens multiples avec cet hypotexte théorique, essentiel et fondateur, que Beyrouth adrénaline révèle le mieux ses choix autant esthétiques que politiques. 
[...] il n'existe pas un seul pays aujourd'hui où l'on puisse se dispenser de réfléchir à la manière de faire vivre ensemble des populations différentes, fussent-elles locales ou immigrées [...] partout se fait sentir la nécessité d'une réflexion sereine et globale sur la meilleure façon d'apprivoiser la bête identitaire. (Maalouf 1998: 43, 203, 204)

9 En outre, sur le plan dramaturgique, l'on repère aisément dans Beyrouth Adrénaline des motifs (la quête existentielle, l'histoire familiale, la guerre, l'exil, le corps, etc.) caractéristiques de l'œuvre d'un autre artiste francophone d'origine libanaise: Wajdi Mouawad. Le dramaturge, qui a vu ses jeunes années deux fois brisées par l'exil ${ }^{4}$, fait de l'écriture un antidote à l'impuissance et au désarroi. Depuis la fin des années 1980, ses productions théâtrales, nombreuses et sans équivalent dans le théâtre d'aujourd'hui, font de la scène contemporaine un lieu de haute intensité émotionnelle. Le théâtre lui est devenu un ancrage face à l'exil: Mouawad creuse, dans une quête d'identité et d'authenticité toujours renouvelée, les répercussions du conflit, pour lui originel, dans le tissu des relations humaines. Alliée à un travail sur la langue française en résonnance avec la langue maternelle oubliée ${ }^{5}$ et une démarche dramaturgique multidisciplinaire proche de la performance, l'aspect politique et sociologique de la démarche du Canadien a enrichi la propre quête de Ghosn. Le déficit communicationnel généralisé entre les individus de la modernité contemporaine omniprésents chez les personnages de Mouawad interpelle profondément Hala Ghosn dans son rapport intime au langage et à la communication. Elle aussi confie ${ }^{6}$ son lien distendu et fragile à l'arabe, qui est la langue maternelle de ses deux parents, puisqu'elle ne parvient pas encore à l'apprendre, comme si le trauma de l'exil l'empêchait de s'approprier cette langue si riche qu'elle admire. Par là, s'explique sans doute le choix de mêler au français de nombreuses répliques en anglais ainsi que des mots et des expressions arabes: un lexique est même fourni en fin de volume (Ghosn 2008 : 74). Bien des dialogues développent ce langage hybridé.

Rima : - Dormir sur le balcon? Majnouna! What about your home in Sri Lanka?

Sandya : - It will be finish for few weeks. There are two floors. One for my brother

and my sister, and one for my fiancé and me.

Rima : - Good, you're lucky. You will be married soon...

Sandya : - Perhaps... no news about your fiancé ?

Rima : - We have a contact, but we don't know where he is. They are probably

waiting un échange avec les autres otages.

Sandya : - Hostages... (Ghosn $2008: 18$ )

Cette écriture polyphonique et polyglotte a aussi pour but de conduire le spectateur francophone au plus près du cœur des Libanais dont l'inclination au plurilinguisme n'a rien de fallacieux. Cette approche linguistique concerne la bande sonore, entièrement dédiée aux classiques arabes : ce sont ces vieux airs des années 1950-1960, de l'Égyptien Abdel Halim à la Libanaise Nour el Houda avec un clin d'œil ironique à Fairouz (Ghosn 2008 : 14, 56, 27) qui passent en boucle dans les appartements des personnages à Paris comme à Beyrouth.

11 Comme Mouawad, Ghosn a la volonté de faire de ses personnages des témoins de l'histoire : Beyrouth Adrénaline se rapproche, de fait, de l'œuvre de la Canadienne Carole Fréchette intitulée Le Collier d'Hélène (2001). Sensibilisée à la situation géopolitique libanaise lors d'une résidence d'écrivain à Beyrouth, Fréchette a conçu une œuvre émouvante et forte dans laquelle la quête d'identité et d'authenticité de son héroïne, déclenchée au cœur de Beyrouth en pleine reconstruction, est le fil directeur de l'action qui passe par la médiation de l'histoire. Chacun des six interlocuteurs beyrouthins d'Hélène va donc partager une expérience de sa guerre, de ses deuils indicibles, de ses 
traumas invisibles et de ses espérances aussi. Leurs compatriotes meurtris, les héros de Ghosn, s'inscrivent évidement dans une semblable lignée de survivants et de résilients.

Dans une société qui s'est éloignée des valeurs tragiques, qui a perdu l'impact de la parole à cause de la pléthore d'images télévisuelles, le théâtre constitue peut-être une chance de redonner un poids et une nécessité aux actes, au langage, au réel. Il apparaît comme une mémoire vivante, qui, au lieu d'occulter les traces de l'événement, valorise au contraire, l'absence, le souvenir, l'écriture, le corps et la mort. (Évrard $2008: 500)$

12 Grâce à ce terreau transdisciplinaire, les concepteurs et artisans du spectacle, dirigés par Ghosn, ont produit une interrogation sur les imbrications entre les histoires individuelles et la grande histoire. Car, en effet, la jeune dramaturge a choisi de ne pas témoigner seule : c'est au sein d'un collectif d'acteurs-auteurs qu'elle évolue depuis toujours. C'est au cœur d'une troupe qu'elle puise les ressources vives qui lui permettent de penser et faire penser le conflit selon la gamme la plus large possible.

\section{Paris-Beyrouth ou Écrire au plateau}

Beyrouth adrénaline est née d'une approche collective fondée sur un jeu d'interrelations complexes entre des artisans du plateau à la recherche de nouvelles relations au texte dramatique et à la scène (Ghosn 2008 : 7). Parce qu'elle mobilise l'ensemble des formes d'expression scénique, l'écriture de plateau se définit par la notion de communauté :

Un homme qui monte sur un plateau, qu'il soit acteur, éclairagiste, maquilleur, technicien, dramaturge, accessoiriste, costumier, truqueur ou scénographe, est à l'évidence un écrivain. Il participe du moins à cette écriture collective que l'on finit par désigner comme œuvre scénique. (Tackels 2015 : 31)

Hala Ghosn et sa troupe pratiquent cet art drôle et tragique, fragmentaire et hybride, cinématographique, autobiographique, qui s'apparente à l'une des tendances majeures du paysage de la dramaturgie contemporaine, héritières du théâtre postmoderne (Pavis 2014 : 77). Elles s'imprègnent d'un modèle dramaturgique singulier, celui de Robert Lepage $^{7}$, dont le dessein est aussi de représenter la figure composite du sujet contemporain. Transitions contrastées, ellipses visuelles et désynchronisations spatiales créent des effets scéniques qui relient Beyrouth Adrénaline à l'esthétique des œuvres du Québécois ${ }^{8}$. En effet, c'est l'approche scénique autant que l'ancrage philosophique qui, dans une pièce comme La Face cachée de la lune (2000), par exemple, offrent à l'artiste d'appréhender la fabrication des identités en tant que création, recherche et compréhension de soi et des autres, qui intéressent Ghosn. L'écrit lepagien parvient à amener sa réflexion à un niveau existentiel qui touche aux étapes de la constitution des individus d'aujourd'hui ; il agit comme un modèle pour Ghosn dans sa recherche scénique autant que dramaturgique et thématique.

Subventionnée par des institutions, l'écriture de plateau de Ghosn requiert une architecture prévisionnelle soigneusement mise au point, suivant les étapes de planification, de formulation et de révision. Avant la publication d'un texte définitif, l'œuvre, toujours en cours d'élaboration, est inscrite dans un processus de création constant. Toujours assorties d'improvisations, les répétitions se poursuivent et décident des choix quant aux aspects textuels et dramaturgiques à conserver. Ce sont ces tâtonnements des avant-textes ${ }^{9}$ qui sont autant d'ajouts, de suppressions, de déplacements, de remplacements qui ne manqueront pas d'intéresser le généticien du texte. 
La pratique de l'écriture de plateau ne diffère pas fondamentalement de celle que les Britanniques nomment à présent devised theatre, un théâtre fabriqué à plusieurs sans distinction nette entre acteur, metteur en scène, dramaturge, scénographe, etc., un théâtre non pas tant de création collective (comme dans les années 1960 et 1970) que de collaboration inter-artistique refusant les trop grandes spécialisations. (Pavis 2014 : 77)

Le dynamisme du protocole de création demeure dissemblable d'un spectacle à l'autre, cependant Ghosn impose son tempo aux étapes obligées. Dans un premier temps, elle œuvre seule en tant qu'auteure, apportant un cadre et une thématique, une première ébauche des personnages, mais aussi des flashes visuels et de la matière théorique ${ }^{10}$ : lectures d'essais, visionnages de documentaires historiques, de films, etc. Le travail à la table se poursuit en collaboration avec Jalie Barcilon ${ }^{11}$. Puis, le binôme assisté de Nicolas Petisoff livre sa trame aux comédiens, "premiers écrivains de plateau, témoins uniques de leur temps, pour leur temps" (Tackels 2015 : 17). Ils sont cinq à incarner sur les planches les huit protagonistes et à considérer le texte originel (qui leur parvient dans une version inachevée) tel un matériau à remodeler en interférence avec leurs corps, leurs énergies et leurs ressentis. Afin d'assurer le meilleur contexte créatif à la troupe, la collaboration se développe donc entre mises au net à la table et improvisations pendant environ huit semaines, selon un séquencier précis. Au fil du texte et du temps, avec son expérience et ses souvenirs, ses intuitions et son intelligence, chaque comédien élabore son personnage à partir des matériaux proposés par les auteurs, conférant au texte dans la vérité de l'instant ici son humour, là son ironie, ailleurs sa cocasserie et surtout son émotion. Le ton est vif et coloré, grave, parfois trivial :

Mona : - Tu sais ce que m'a dit François, le frère de Marie-Laure? Qu'en tant que

Libanaise, je suis potentiellement une terroriste [...]

Sandya : - I don't want to die ! I don't want to die!

Najat: - Shut your mouth! Shut your mouth!

Rima (à Sandya) : - Please, Sandya, on travaille demain. We need to sleep. (Ghosn

$2008: 12,43)$

17 Aucun pan de la mise en scène n'est préétabli et la démarche scénographique prend corps peu à peu au rythme de l'appropriation par les acteurs de l'espace et des personnages. De fait, les acteurs n'arrivent jamais en répétition avec un texte clé en main: l'écriture s'élabore en cours de mise en scène, de façon très instinctive. C'est au plateau que s'opèrent ajouts, coupures et repentirs du groupe ; c'est donc de cette dynamique que naît l'esthétique du montage, totalement cinématographique dans le sens où elle repose sur un travail de coupes, d'ellipses et de superpositions. La syntaxe scénique repose sur une esthétique du fragment thématique (alternance de lieux, sauts temporels) et du fondu enchaîné (jeux de lumière et jeux sonores). La construction englobante de Beyrouth adrénaline en alternance de scènes de Paris et Beyrouth (six scènes sont à Paris, cinq à Beyrouth ; une séquence mime une connexion téléphonique entre les deux villes) permet de dire la détresse qui afflige ceux qui ne vivent pas la guerre et ceux qui subissent puisque la scène est divisée en trois parties : deux blocs surélevés font office de balcons, où se déroulent les scènes beyrouthines; ils encadrent un autre espace au niveau du sol où ont lieu les scènes parisiennes. Dès les prémices du spectacle, l'équipe artistique travaille étroitement avec l'équipe technique car les textes hybrides font usage de matrices plastiques, chorégraphiques et transdisciplinaires : du récit originel, simple et touchant, se dégage l'esprit du temps tissé d'altérité et de virtuel. L'optique scénaristique du collectif, emmené par Ghosn et son collaborateur artistique Nicolas Petisoff avec l'assistance du créateur son et vidéo Frédéric Picart, repose sur un ancrage réaliste 
immersif établi avec des succès arabes langoureux, des bribes d'émissions radiophoniques et des extraits d'une retransmission télévisuelle d'un match de football, tant et si bien que «le plateau avale tous les médiums » (Tackels $2015: 18$ ).

VOIX OFF TÉLÉ: [...] les otages français détenus au Liban, Marcel Carton, Marcel Fontaine, Michel Seurat, Jean-Paul Kaufmann et l'équipe d'Antenne 2, Philippe Rochot, Georges Anselme, Aurel Cornéa et Jean-Louis Normandin, n'ont toujours pas été libérés, cela fait très exactement... (Ghosn 2008 : 28)

Par conséquent, c'est bien dans un esprit collectif aussi alerte que dynamique que la troupe entière, sans jamais se départir de sa profondeur, puise le détachement et la légèreté seules aptes à créer des situations émouvantes et drôles, de celles qui amènent les spectateurs à réfléchir au passé récent, à leurs propres choix en tant que citoyen et, dans un élan de solidarité, à s'interroger sur ce qu'ils auraient fait à la place des personnages, et finalement, peut-être, à sentir la révolte les envahir telle une montée d'adrénaline salvatrice.

\section{Une œuvre dramatique saisie par l'histoire}

La force des écritures de plateau tient à ce que le spectateur lui-même devient partie prenante du processus d'écriture; il n'est plus seulement récepteur d'une vision du monde, fût-elle d'une actualité brûlante et immédiate de sa contemporanéité. Le travail dramaturgique ne s'achève que dans cet ultime partage. «Le dernier écrivain du plateau est bien le spectateur » (Tackels 2015 : 63). Selon l'étymologie, le théâtre est justement «le lieu d'où l'on regarde » et la problématique du traitement de la contemporanéité et du regard posé sur la réalité est habilement exploitée dans Beyrouth adrénaline, où il est finalement moins question de l'ancrage historique que de la fonction narrative cognitive, anthropologique du texte littéraire.

La pièce conduit en effet à une pensée du pouvoir de la représentation; elle plonge d'abord le spectateur au cœur de situations quotidiennes, ordinaires pour les habitants de Beyrouth: coupures d'eau intempestives désorganisant les tâches ménagères et empêchant les soins corporels (Ghosn 2008: 16-17), couvre-feu et nuits passées entre voisins dans les caves de fortune des immeubles pour se protéger des bombardements (Ghosn 2008: 42-43), crises hystériques (Ghosn 2008: 42, 58), incompréhensions et disputes comme autant de reprise individuelles des tensions qui se jouent au niveau collectif. La guerre du Liban est un conflit civil né d'un imbroglio de divergences inter- et intracommunautaires et ponctuée d'interventions étrangères dont les séquelles sont encore douloureuses pour les citoyens : à côtés des pertes humaines qui s'évaluent à plus de 250000 victimes civiles, des déplacements de populations (localement ou vers l'étranger avec la vague de réfugiés, de rapprochements familiaux et de demandeurs d'asile), de la reconstruction infrastructurelle et politique longue et laborieuse, le maintien en place de milices armées autonomes et la reprise ponctuelle des violences prolongent sur presque deux générations désormais la désorganisation nationale qui est encore aggravée par la non-résolution du problème palestinien. Sur scène, la guerre demeure cependant sans nom et avant la clausule parisienne, lorsque se tient la conférence de Zyad, les circonstances du déclenchement du conflit armé ne sont jamais évoquées; aucune mention n'est jamais faite d'un chef politique ou d'un groupe de combattants, à peine est-il question d'une milice, de tireurs embusqués sur les toits. La guerre demeure dans le non-dit, totalement indicible mais envahit la scène jusqu'à 
l'asphyxie. La possibilité pour l'histoire récente d'être saisie par la fiction est en revanche offerte par un vaste questionnement sur la mémoire nationale et ses lieux patrimoniaux. Suivant le dessein de cerner les "Origines d'un conflit multiséculaire », Zyad remonte le cours de l'histoire sur plus de 4000 ans y cherchant en vain les causes du conflit, conscient qu'elles résident dans le cœur des hommes depuis des temps immémoriaux :

Des fouilles archéologiques révèlent, au cœur de la ville moderne de Beyrouth, sous l'actuelle place des Martyrs, la présence de vestiges d'habitations datant du néolithique, ainsi que d'innombrables installations à ciel ouvert, datant du mésolithique. Les plus anciennes empreintes humaines découvertes remontent au paléolithique, c'est-à-dire en -600 000 ans avant notre ère. Elles se situent dans ce qui deviendra plus tard le quartier de Ras Beyrouth, et qui n'est alors qu'une île... Pour autant peut-on attribuer l'entière responsabilité de ces événements à la seule présence humaine dans la région. (Ghosn $2008: 72$ ) des certitudes factuelles et circonscrites; dans le même temps, elle déplace l'émotivité générée par l'actualité libanaise, dont les médias nous abreuvent (reportages, tables rondes et débats). La combinaison de l'histoire, de l'actualité et de la fiction dans Beyrouth adrénaline se fonde alors sur un entre-deux où pénètre l'inavouable, le non visualisable qui amène à une plus large compréhension des événements. L'expérience de la guerre devient le creuset d'une altérité radicale qui sépare les Beyrouthins des Parisiens : elle est le facteur de différenciation des protagonistes entre eux, la source de leur incommunicabilité fondamentale, puisqu'elle souligne le clivage entre initiés et noninitiés. Face au caractère paroxystique des expériences que le conflit armé détermine, les réactions de Mona et de Zyad qui oscillent entre désir d'action et culpabilité inhibante, gèrent un vécu frappé d'incomplétude: confrontés à l'intensité du vécu de leur frère Marwan, qui n'a pour avenir que l'exil (option suivie par Zyad) ou l'engagement meurtrier (option suivie par Touffic), ils ne peuvent plus habiter avec naturel leur propre existence. Au contact de la réalité libanaise, la (relative) facilité de leur quotidien de lycéenne pour l'une, et d'universitaire pour l'autre, apparaît comme l'origine du vide existentiel, de la précarité de leur sentiment d'être. De la sorte, Mona décide de se rendre sur place afin d'expérimenter par elle-même son rapport au Liban, sa terre natale : à fleur de peau, la jeune femme souhaite se confronter au fantasme d'un pays idéal qu'elle entretient depuis son exil français sous l'influence de son aîné. «J'ai envie de savoir, j’ai besoin de savoir » (Ghosn $2008: 35$ ).

Symétriquement, Marwan et sa voisine Rima évoluent dans un présent essentiellement absurde parce qu'il est sans projet et qu'il n'y règne que l'instantané de la guerre et de ses brutales destructions. L'existence des habitants sous une telle tension confinant à la folie est pleinement incarnée par la vieille Najat, qui vit repliée dans le grand âge, dans une existence désormais sans chronologie, la maladie signalant une fuite hors de la réalité présente. Sur scène, la ville de Beyrouth devient un lieu de mémoire ${ }^{12}$ qui possède pour l'ensemble des protagonistes une charge intellectuelle, identitaire, symbolique et affective forte, la mémoire étant fortement spatialisée. Et Najat est le personnage qui figure le capital mémoriel collectif du Liban qui n'est plus vécu spontanément en raison des destructions patrimoniales: elle revit en les confondant les différentes époques historiques. "Si c'est pas les Italiens, c'est les Turcs? On est attaqués par les Turcs, Marwan? Par ces Hayawen, ces Ottomans?» (Ghosn 2008 : 40). Pour Mona, Beyrouth, la ville de sa naissance, est un lieu autant qu'un lien de mémoire : si elle valorise le passé tel un âge d'or, c'est afin d'y trouver des repères et de recréer du vivre-ensemble 
aujourd'hui. La dernière scène sans paroles la laisse sur le balcon s'installant pour une séance de bronzage, la chaleur du soleil sur la peau signifiant la réintégration de son être dans un biotope depuis longtemps quitté.

Par ses lignes de fuites, l'œuvre souligne encore que le lien à la mémoire nationale n'est jamais inné ; aussi, lorsque Sophie évoque le Liban, elle entremêle les topoï immatériels, physiques, culturels et spirituels de la mémoire collective qui certes expriment son désir de connaitre la civilisation libanaise, mais dont ressort surtout une maladroite compilation de stéréotypes: c'est dans l'espace et par l'espace que cette mémoire se forge :

Qu'est-ce qu'il faut que je fasse pour exister? Que je me foute à poil !!! Je rentre plus dans mes fringues. J'ai pris deux kilos avec leurs plats libanais! Je craque! [...] Je suis pieds nus en plus, à cause de leurs tapis à la con! J'en ai marre! Je vais me voiler, voilà ! [...] Je fais plein d'efforts, j'ai appris tous les noms par cœur : Tripoli, Camille Chamoun, Walid Jomblat, fedayin, Maronites, Maamoul, Chiites, shit ! Mais j'imprime pas. Je suis vide, personne ne me voit, Sophie la petite Française invisible. (Ghosn 2008: 53)

La présence de l'employée sri-lankaise anglophone, apeurée, totalement dépassée par les événements de ce pays qu'elle ne comprend pas généralise l'absurdité de la situation. Tout son être signale que cette guerre n'est pas « sa » guerre et que le soleil du Liban, qui plaît tant à Rima, lui est un autre exil.

Avec cette « reconstruction hésitante et inquiète d'expériences partielles, habitée par une double question: comment en est-on arrivé là ? L'homme a-t-il encore un quelconque avenir?» (Viart et Vercier 2008: 130), Ghosn concentre dans son travail des interrogations philosophiques sur les conséquences de la guerre civile, sur les effets psychologiques et sociaux de l'exil et sur la question du legs et de la transmission. Car, c'est bien dans la rencontre avec l'histoire de l'autre que se dévoile la fragilité du sens des existences de chacun. Et avec Beyrouth adrénaline, Ghosn a justement le dessein de faire en sorte qu'en toute fraternité, les Libanais expriment enfin sur le mode cathartique la palette de sentiments et de désirs qui sont autant de pulsions vitales et d'anéantissement qui les assaillent.

Conçu tel un espace de réflexion collective sur l'individu dans l'histoire, Beyrouth adrénaline pose ainsi avec beaucoup de sincérité la question des enjeux de la mise en fiction du passé récent. Hala Ghosn y fictionnalise sa bi-nationalité problématique. « C'est notre regard qui enferme souvent les autres dans leurs plus étroites appartenances, et c'est notre regard aussi qui peut les libérer " (Maalouf 1998: 32). Nourrie par un théâtre en prise avec le temps, celui de Robert Lepage ou de Wajdi Mouawad, la dramaturge parvient à son tour à lier le soi et l'autre, le passé et l'avenir, l'histoire collective et la mémoire individuelle, le temps et l'espace, et finalement l'universel et l'intime. C'est assez dire que, née au plateau et transcendée par un regard original sur l'autre, cette pièce moderne et insolite s'avère tout à fait apte à enrichir notre compréhension des représentations littéraires de la guerre civile libanaise. 


\section{BIBLIOGRAPHIE}

Boucheron, Patrick, 2011, « On nomme littérature la fragilité de l'histoire », Le Débat, nº 165, p. 41-56.

Compagnon, Antoine, 2011, « La crise des disciplines », Le Débat, $\mathrm{n}^{\circ}$ 165, p. 62-70.

Évrard, Fabrice, 2008, « Le théâtre en prise sur le monde », dans D. Viart et B. Vercier (dir.), La Littérature au présent. Héritage, modernité, mutations, Paris, Bordas, p. 499-515.

Féral, Josette, 2011, « Pour une génétique de la mise en scène. Prise 2 », dans Théorie et Pratique du théâtre : Au-delà des limites, Montpellier, L'Entretemps, p. 64-77.

Gengembre, Gérard, 2011, « Histoire et roman aujourd'hui : affinités et tentations ", Le Débat, n 165 , p. 122-135.

Grésillon, Almuth, 1994, Éléments de critique génétique. Lire les manuscrits modernes, Paris, PUF.

Lepage, Robert, 2007, La Face cachée de la lune, Québec, L’instant même/Ex Machina, coll. «L'instant scène ».

Maalouf, Amin, 1998, Les Identités meurtrières, Paris, Grasset.

Mouawad, Wajdi, 2008, Seuls. Chemin, texte et peintures, Arles, Actes Sud.

Nora, Pierre, 2011, « Histoire et roman : où passent les frontières ? ", Le Débat, nº 165, p. 6-12.

Pavis, Patrice, 2002, Dictionnaire du théâtre, Paris, Armand Colin.

Pavis, Patrice, 2014, Dictionnaire de la performance et du théâtre contemporain, Paris, Armand Colin.

Tackels, Bruno, 2015, Les Écritures de plateau : états des lieux, Besançon, Les Solitaires Intempestifs.

Viart, Dominique et Vercier, Bruno (dir.), 2008, La Littérature au présent. Héritage, modernité, mutations, Paris, Bordas.

\section{Fuvre étudiée}

Ghosn, Hala et Jalie Barcilon, 2008, Beyrouth adrénaline, Bruxelles, Lansman.

\section{NOTES}

1. Ce dont l'attribution du prix Nobel au romancier Patrick Modiano, en 2014, atteste également.

2. Dans un autre registre, l'on pourrait aussi citer le succès populaire de la farce politique mettant en scène Talleyrand et Fouché, Le Souper (1989) de Jean-Claude Brisville, ou celui des Palmes de Monsieur Schutz (1989) de Jean-Noël Fenwick, une pièce centrée sur le couple de physiciens Pierre et Marie Curie.

3. Elle a été publiée aux éditions Lansman en Belgique en 2008.

4. Né en 1968, Mouawad fuit la guerre du Liban avec sa famille à l'âge de huit ans; il émigre en France avant de s'expatrier au Canada en 1983. 
5. Les acteurs que Mouawad dirige doivent adopter les intonations de l'arabe, ainsi qu'il l'explique dans Lise Lenne, « Entretien avec Wajdi Mouawad », Agôn, Entretiens et documents, [En ligne], mis à jour le 21 octobre 2008, http://agon.ens-lyon.fr/index.php?id=290.

6. Lors de la Table ronde «Femme et francophonie: pourquoi j'écris, pourquoi je crée en français ", tenue à l'Université du Koweït, le 24 mars 2015.

7. Metteur en scène, scénographe, auteur dramatique, acteur et cinéaste, Robert Lepage (né en 1957) se réfère constamment à son expérience cinématographique, et l'applique dans son travail théâtral et, s'il met en scène des œuvres originales, il innove notamment par l'utilisation de nouvelles technologies.

8. L'influence est flagrante et Ghosn l'a du reste reconnue dans une correspondance personnelle datée du 8 août 2015.

9. C'est la critique Almuth Grésillon (1994: 214) qui donne le nom d'«avant-texte » à tous les documents de cette phase de la gestation d'un spectacle.

10. Cet attrait pour les sujets documentaires constitue l'une des caractéristiques du théâtre d'aujourd'hui et les transmodalisations y sont fréquentes.

11. Née en 1978, Jalie Barcilon est auteure et metteure en scène (Art'Catastrophe, 2005 et Le Deuxième Fils). Elle a co-écrit Beyrouth adrénaline et Apprivoiser la panthère. En 2010, elle a mis en scène Just like a woman. Elle anime aussi des ateliers d'écriture et de mise en scène.

12. Les Lieux de mémoire est un ouvrage collectif en sept volumes rassemblant plus d'une centaine d'auteurs, publié sous la direction de Pierre Nora entre 1978 et 1992 dont l'ambition est de recenser les principaux monuments, lieux et symboles qui contribuent à forger l'identité culturelle française à travers l'histoire. Ces «lieux » au cœur de la mémoire nationale peuvent évidemment être matériels ou des idéels.

\section{RÉSUMÉS}

Cet article propose l'analyse d'une œuvre dramatique actuelle dans ses liens avec l'histoire : dans un premier temps, il met en lumière le modus scribendi original d'une pièce saisie par un événement historique contemporain (la guerre du Liban) et ancrée dans le parcours intime de son auteur, la Franco-Libanaise Hala Ghosn (née en 1976) ; il inscrit dans un second temps cette œuvre selon ses particularités dans la constellation des écritures de plateau afin, dans un troisième temps, d'expliciter les défis anthropologiques qu'elle soulève.

This article offers an analysis of a dramatic work in its current links with History: at first, it highlights the original modus scribendi of a work written about a contemporary historical event (the Lebanese war) and rooted in the intimate path of Franco-Lebanese author, Hala Ghosn (born in 1976). In a second part, this article places the text with his feature in the nebula of current devised theater, in order to clarify the anthropological challenges it raises in a third part.

\section{INDEX}

Mots-clés : théâtre contemporain, écriture de plateau, histoire, guerre du Liban Keywords : contemporary theater, devised theater, history, Lebanon war 


\section{AUTEUR}

\section{ISABELLE BERNARD}

Université de Jordanie 